\title{
Recurrent astrocytoma in a child: A report of cytogenetics and TP53 gene mutation screening ${ }^{1}$
}

\author{
Anke Dam, Johanna M. Fock, Vanessa M. Hayes, Willemina M. Molenaar, ${ }^{2}$ \\ and Eva van den Berg
}

Departments of Pathology (A.D., W.M.M.), Child Neurology (J.M.F.), and Medical Genetics (E.v.d.B., V.M.H.), University Hospital of Groningen, The Netherlands

An 8-year-old girl presented with a cerebral tumor and 3 recurrences within 15 months. The primary tumor was a low-grade astrocytoma, but the recurrences showed progressively malignant phenotypes with increasing mitotic activity and MIB-1 labeling indices. Radiotherapy was given between the first and the second recurrences. Cytogenetic analysis of the first and the second recurrences showed abnormal karyotypes. There seemed to be 2 common breakpoints in these 2 recurrences. TP53 gene mutation screening, using comprehensive denaturing gradient gel electrophoresis, revealed among others a possibly causative mutation of exon 5 in 3 of 4 tumor samples. The meaning of TP53 mutations in low-grade astrocytomas is still unclear, but the highly abnormal karyotypes, which are unusual in these tumors, probably provide genetic evidence for the unexpected aggressive behavior of the tumor in this patient. Neuro-Oncology 2, 184-189, 2000 (Posted to Neuro-Oncology [serial online], Doc. 00-013, May 16, 2000. URL <neuro-oncology.mc.duke.edu>)

$\mathrm{T}$ 1 he most common histologic type of astrocytic tumor in children is the pilocytic astrocytoma, but diffuse astrocytomas of all grades of malignancy are observed as well (Giannini and Scheithauer, 1997). They have a predilection for the cerebellum and brainstem, but may occur elsewhere in the CNS (Cohen and Duffner, 1994). Outcome after surgery is in most cases rather

Received 24 February 2000, accepted 29 February 2000.

${ }^{1}$ This study was supported by the Pediatric Oncology Foundation, Groningen, The Netherlands.

${ }^{2}$ Address correspondence and reprint requests to W.M. Molenaar, Department of Pathology, University Hospital of Groningen, Hanzeplein 1, 9713 GZ Groningen, The Netherlands.

${ }^{3}$ Abbreviations used are as follows: DI, DNA index; FISH, fluorescence in situ hybridization; LI, labeling index. favorable (Lapras et al., 1986; Sgouros et al., 1995). Cystic astrocytomas often can be resected totally and seldom recur. Depending on the grade and classification of the tumor, radiation therapy may be added (Lapras et al., 1986). Sometimes the biologic behavior of these astrocytic tumors is rather unpredictable, and follow-up of these patients is always necessary. In the case of tumor recurrence, a second-look surgical intervention is needed.

The current study describes the histology, including MIB-1 labeling, cytogenetic findings, and the results of TP53 mutation analysis, in a patient with a peculiar clinical course and unusual histology.

\section{Clinical History and Histology}

The patient, an 8-year-old girl, was born without difficulty after an uncomplicated pregnancy. Motor milestones and mental development were normal until the age of 5 . From then on, she developed an impulsive and unpredictable behavior, and at age 6 she had school problems. She started to complain about headache, nausea, and vomiting when she was 8 years old. The clinical history was completed with complaints of paresthesia in her left arm, painful eyes, and headtilting. Neurologic examination revealed a torticollis to the right, vision loss, and papilledema. MRI of the brain showed a multilocular cystic mass in the left frontal lobe with edema, a shift to the right of the medial structures, compression of the third ventricle, and a slight hydrocephalus. A subtotal resection of the tumor mass was done. Histologically, the specimen showed 2 different components. One consisted of microcystic tumor tissue with low cellularity, compatible with low-grade astrocytoma (Fig. 1A). The second component consisted of a more cellular, solid proliferation of medium-sized round cells with little pleomorphism (Fig. 1B). There was infiltration in the overlying cortex and meninges. There was virtually no mitotic activity. The MIB $\mathrm{LI}^{3}$ was 0.17 . 

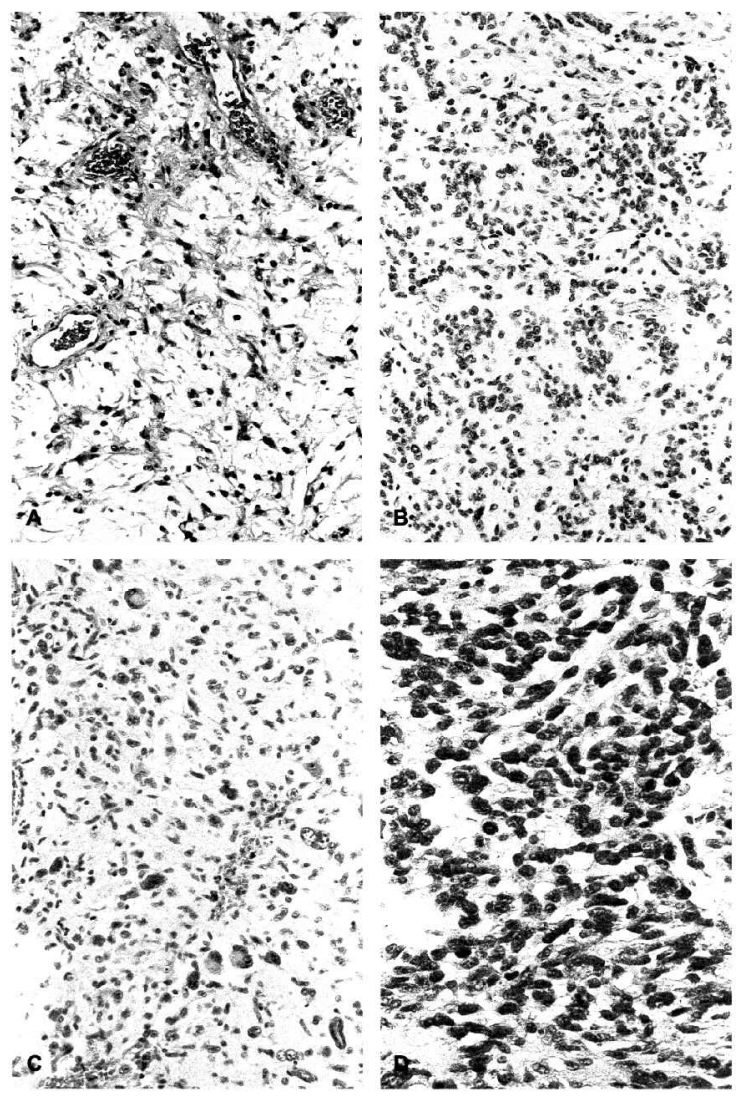

Fig. 1. Histology of the primary tumor showing areas with low (A) and higher (B) cellularity; second recurrence, after radiotherapy (C), and third recurrence showing undifferentiated tumor tissue (D). Hematoxylin and eosin. A, B, and C, original magnification X200; D, original magnification $X 400$.

Necrosis was focally present, primarily in the area with low cellularity. A diagnosis of low-grade astrocytoma with focally increased cellularity was made.

After surgery, the patient developed cognitive problems, mostly involving verbal expression and dyslexia, but without distinct aphasia. She suffered from anxiety problems and complained about paresthesias in the left arm. Followup MRI showed the resection cavity with enhancement of gadolinium at the edges, but without evidence for tumor recurrence (stable disease). Six months postoperatively, complaints of vomiting and headache recurred, and clinical examination revealed papilledema again. MRI showed a mass in the subdural space and intraparenchymally in the left frontal lobe. A second-look craniotomy was done, and a hemorrhagic tumor mass was resected. Histologic examination showed areas similar to the initial tumor (coded Rec. 1a). In addition, areas with high cellularity and nuclear atypia were observed (coded Rec. 1b), with mitotic activity up to $8 / 2 \mathrm{~mm}^{2}$, whereas the MIB LI had increased to 2.35 , both indicating progression in malignancy. Radiation therapy was started: 59 Gy in 33 fractions, shrinking field. Within 4 months the complaints recurred, and MRI showed an increasing volume of the frontally localized cerebral cyst and enhancement of gadolinium in its wall. Aspiration of cyst fluid showed a very high protein count of $46 \mathrm{~g} /$ liter. An Ommaya reservoir was placed, and repeated aspirations were done to diminish the volume of the cyst. One year after the initial presentation, the cyst-plus-tumor mass was resected again. This second recurrence (coded Rec. 2), after radiation therapy, showed more pleomorphism and multinucleation of tumor cells, but no mitotic activity (Fig. 1C). The MIB LI was 0.21 , in the range of the first tumor sample.

Despite the resection, the patient developed a progressive frontal syndrome. A CT scan showed an enlarging cyst with increased enhancement and progression of tumor mass, compression of the foramen of Monro, and obstructive hydrocephalus. A fourth surgical exploration was done with resection of a well vascularized tumor mass (coded Rec. 3) out of the cyst wall, and a ventriculoperitoneal drain was placed. The histology, 15 months after the initial diagnosis, showed densely cellular, undifferentiated tumor tissue (Fig. 1D) with brisk mitotic activity (up to $23 / 2 \mathrm{~mm}^{2}$ ), a MIB LI of 8.59 , and extensive infiltration in the meninges. Within a month, identical complaints of headache and behavioral disturbances reappeared. No adequate curative therapy could be given any more. The patient died 4 months later. Permission for autopsy was refused.

\section{Materials and Methods}

All tumor samples were stained for MIB-1 (see below). Samples of the first (Rec. $1 \mathrm{a}$ and $1 \mathrm{~b}$ together) and the second recurrence (Rec. 2) could be processed for tissue culture, cytogenetics, FISH, and DNA flow cytometry. In addition, all tumor samples except the third recurrence could be screened for mutations in the TP53 gene.

\section{MIB-1 Labeling}

MIB-1 labeling was performed on formalin-fixed paraffin-embedded tissue of all the resection material. Each section measuring $5 \mu \mathrm{m}$ was cut, floated in a water bath of $50^{\circ} \mathrm{C}$, and mounted on aminopropylethoxysilane (APES)-coated slides, which were placed at $60^{\circ} \mathrm{C}$ for $15 \mathrm{~min}$ and dried overnight in an oven at $37^{\circ} \mathrm{C}$. Wet autoclaving was used twice for antigen retrieval. The slides were then incubated with primary MIB-1 antibody (Immunotech, Villeponte, France) diluted 1:400. Two-step immunostaining was performed using antimouse biotin and alkaline phosphatase-conjugated streptavidin, according to the manufacturer's procedure of the Biogenex kit (Biogenex, San Ramon, CA). NBT/BCIP (4-nitroblue tetrazolium chloride and bromo-chloro-indolyl-phosphate; Boehringer Mannheim, Mannheim, Germany) was used as substrate. Ten high-power $(\times 400)$ microscopic fields were randomly selected from each tumor slide, and the cells were counted. MIB-1 LI was calculated as the number of positive cells divided by the total number of cells.

\section{Cytogenetics}

For cytogenetic analysis, part of the tissue was minced with scalpels, incubated in a collagenase-deoxyribonuclease solution, and cultured in RPMI 1640 supplemented with 
Table 1. Karyotype description of the first and the second recurrence

First recurrence (Rec. $1 \mathrm{a}+\mathrm{b}$ together):

43 45,XX,-18[cp3]/40, XX, add(1)(q21), del(4)(q21q31),-5,-13,-14,der(14)t(?9;14). ish add(14)(p13)(wcp9-,wcp14+),-15,-17,-18,-21, $-22,+2 \operatorname{mar}[1] / 79 \sim 80$, idem $\times 2,-\operatorname{del}(4)(q 21 q 31),+14,-\operatorname{der}(14) t(? 9 ; 14)$.ish add(14)(p13)(wcp9-,wcp14+), +21,-2mar[cp2]/46,XX[19] .

Second recurrence (Rec. 2):

44 46,X, del(X)(q23), del(2)(p13), add(3)(q29), add(6)(q21), der(14)t(?9;14).ish t(14;14)(p13;q23)(wcp9-,wcp14+,D14S308++), add(15)

(q24),t(17;18)(q11;p11.3), dd(17)(q22), add(22)(q13) [cp7]/43 46,XX,t(1;7)(q21;p22) del(7)(q32),t(5;8)(p15;q13), add(8)(q13),t(11;16) (q13;p12), inv(12)(q13q15), del(16)(q21)[cp2]/46,XX, del(6)(p22)[cp2].

$16 \%$ fetal calf serum, glutamine, and antibiotics. After short-term culturing, the cells were harvested, and chromosome preparations were made according to standard cytogenetic techniques. The chromosomes were G-banded using trypsin/pancreatin, and karyotypes were described according to ISCN (Standing Committee on Human Cytogenetic Nomenclature, 1995).

\section{DNA Flow Cytometry}

For DNA flow cytometry, we used a method developed by Vindelov et al. (1983). Nuclei were isolated using a detergent-trypsin method, exposed to ribonuclease, and incubated with propidium iodide. The intensity of the fluorescent signal was measured using a Coulter Epics Elite flow cytometer (Coulter, Hialeah, FL). For each sample, 30,000 events were analyzed.

\section{FISH}

For FISH studies, specific libraries for chromosomes 9 and 14 (commercially available from Cambio, Downer's Grove, IL) and the Tel 14q DNA probe (P5428-DIG; ONCOR, Gaithersburg, MD) were used. In situ hybridization was carried out essentially according to the manufacturer's protocol.

\section{TP53 Gene Analysis}

For genetic analysis of the TP53 gene, DNA was extracted from $5 \times 10 \mu \mathrm{m}$ sections of paraffin-embedded tumor tissue using the chelex boiling method (Stein et al., 1992). The entire coding region, including all splice site junctions, of the TP53 gene was amplified in 12 amplicons using nested polymerase chain reaction primers under conditions previously described (Hayes et al., 1999a, 1999b). All amplicons were electrophoresed in a $9 \%$ polyacrylamide gel containing $5 \%$ glycerol and a $35 \%$ to $75 \%$ UF denaturing gradient $(100 \%$ UF $=7 \mathrm{M}$ urea/40\% deionised formamide). Electrophoretic conditions used were as described elsewhere (Hayes et al., 1999a, 1999b).

\section{Results}

Results of the cytogenetic analysis are described in Table 1. Figs. 2 and 3 show karyotypes of Rec. $1 \mathrm{a}$ and b, and Rec. 2, respectively. In Rec. 1a and b, only one aberrant cell, 40,XX, could be karyotyped. However, 2 more cells 79 80, idem $x 2$, were found, indicating that most of the aberrations in the first cell were clonal. In addition, Rec. 2 showed many nonclonal numerical and structural aber-
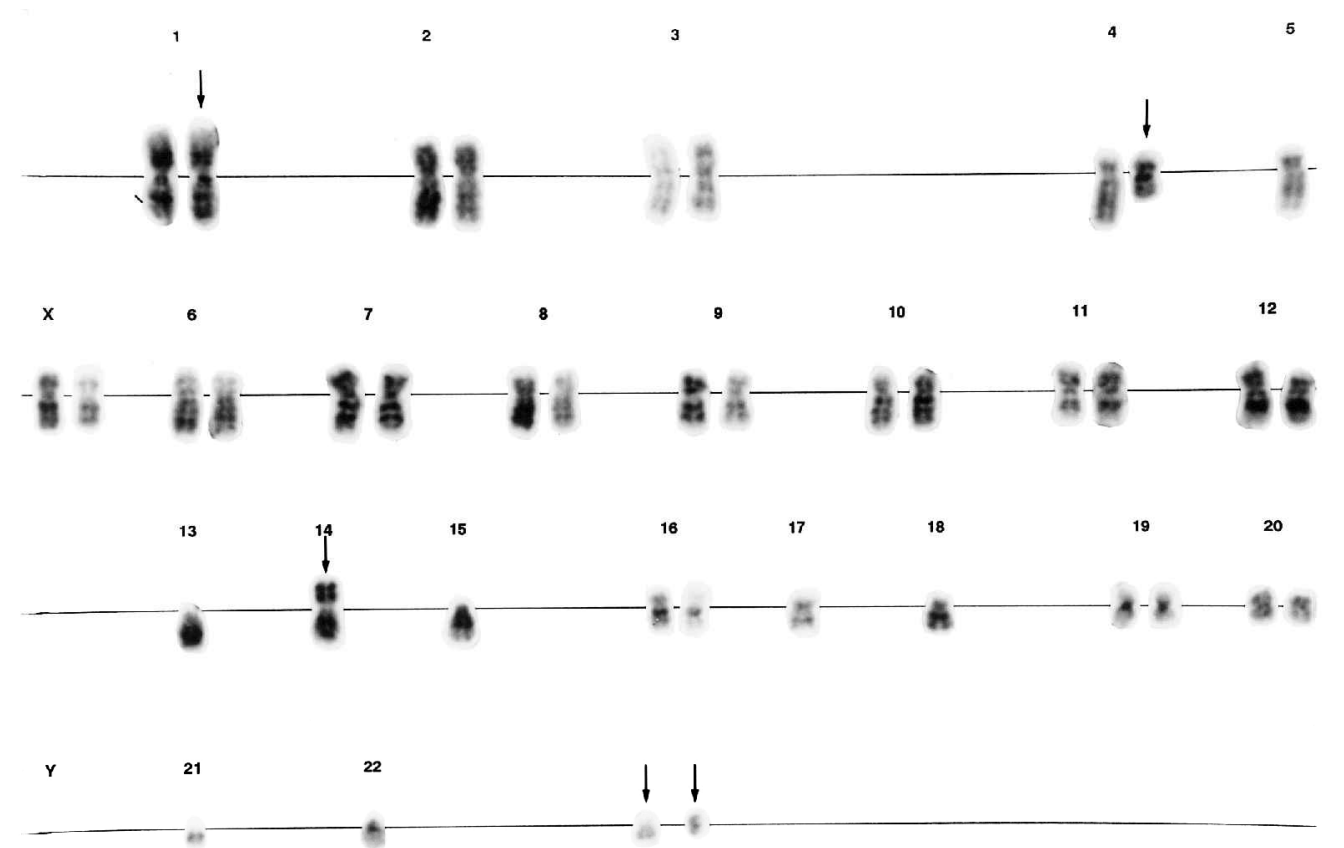

Fig. 2. Representative karyotype of the first recurrence. 

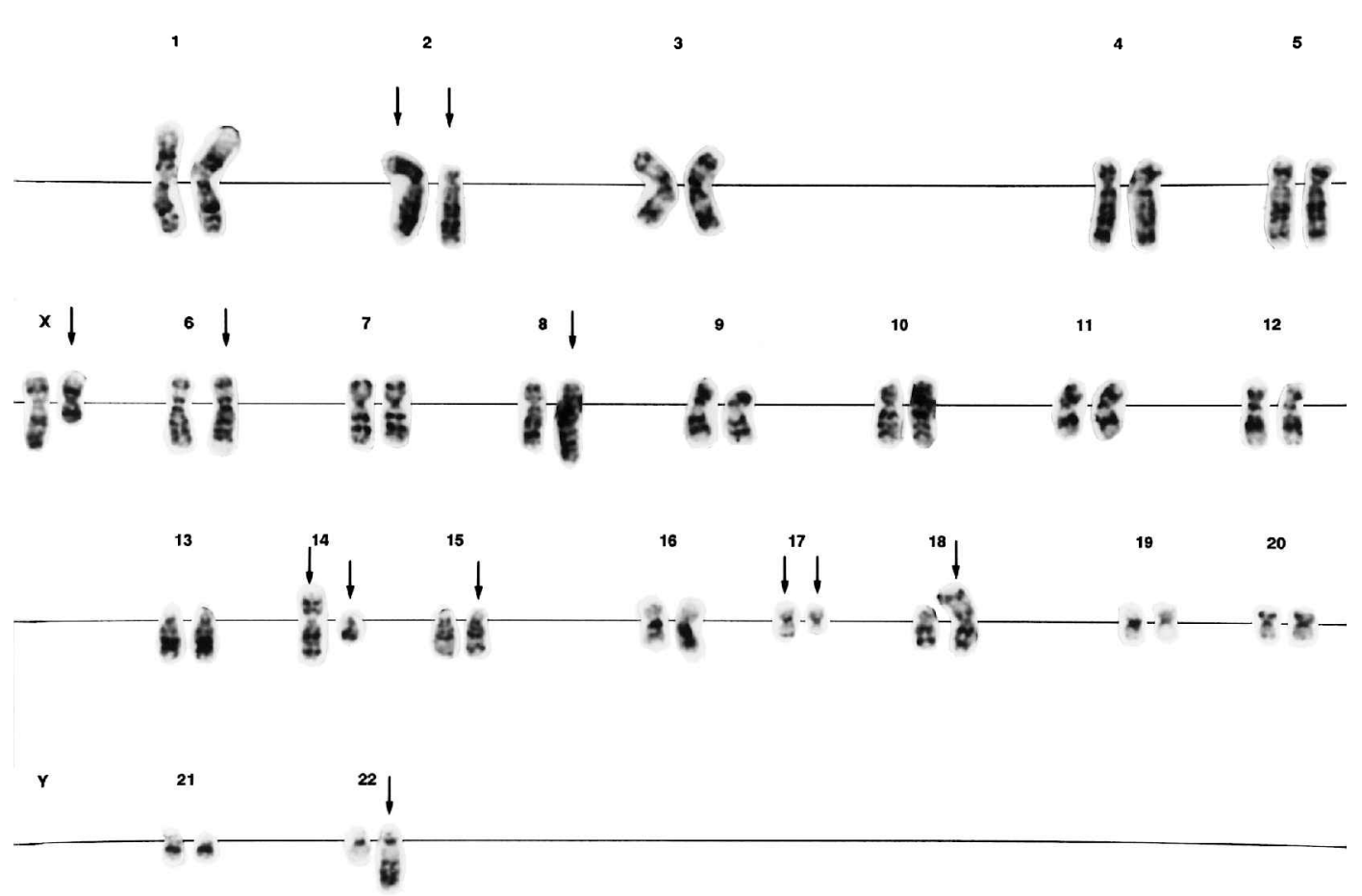

Fig. 3. Representative karyotype of the second recurrence.

rations, typically chromosomal rearrangements of the balanced type.

Despite the radiation therapy, 2 breakpoints seemed to be common to both karyotyped recurrences: 1q21 and $14 \mathrm{p} 13$. Derivative chromosomes revealing the $1 \mathrm{q} 21$ breakpoints were not identical, but the structural rearrangement involving $14 \mathrm{p} 13$ seemed similar in both cases. FISH analysis revealed the presence of a $t(14 ; 14)$ in Rec. 2. However, this was not detected in Rec. 1a and b.

The DNA flow cytometry histograms of both Rec. 1a and $1 \mathrm{~b}$ revealed a hypertriploid peak (DI 1.75 and 1.78 ), which is in agreement with the clone of 79 80 chromosomes. Cells with 40 and 43 45 chromosomes were present in low numbers and were in the diploid range, probably explaining why they were not detected in the DNA histogram as a peak separate from the normal diploid peak. In Rec. 2, however, the DNA flow histogram demonstrated an additional tetraploid peak (DI 1.95), whereas the karyotype showed merely 39 45 chromosomes. This might be the result of a growth advantage in tissue culture of cells with low chromosome numbers, or a sampling error with regard to which part of the tumor had been cultured.

TP53 mutations found are depicted in Table 2. A possibly causative mutation (Arg156-Pro) was found in the initial low-grade tumor, which persisted to Rec. 1b, the more malignant form of the first recurrence. This exon 5 mutation resulted in a nonconserved amino acid change within the DNA binding domain of the p53 protein. As depicted in Fig. 4, this mutation occurred hemizygously/homozygously in Rec. $1 \mathrm{~b}$, but heterozygously in the primary tumor and Rec. 2. This may be explained by contamination from normal cells. An additional, possibly noncausative intronic mutation (intron 9) was found in the primary tumor and Rec. 1b. This mutation did not reappear after radiotherapy. According to the NetGene2 prediction program (Hebsgaard et al., 1996), this mutation does not affect the splicing of intron 9. In Rec 1a, no causative exon 5 mutation or intron 9 mutation was found. However, an additional possibly causative mutation (Thr211-Ile) in exon 6 of the TP53 gene resulting in a nonconserved amino acid change was found, suggesting that it may have arisen as an independent tumor in this patient. After radiotherapy (Rec. 2), the causative exon 5 mutation was observed, the intron 9 mutation was lossed, and an additional intron 2 mutation involving a one-base-pair insertion, and presumably having no effect on the p53 protein, was also found. Unfortunately, Rec. 3 did not give any interpretable results.

\section{Discussion}

Astrocytomas in children are regarded as benign tumors and, after resection, no radiation therapy is given. It is known that after a total resection, recurrences hardly occur. The recurrence of tumor in low-grade astrocytomas is at the primary site or, when radiation therapy is given, in the area of the field of radiation (Dirks et al., 1994). Clinical parameters are important when considering the risk of recurrence in a patient with a low-grade astrocytoma, and regulation of cell proliferation and genetic abnormalities also may be of importance to expectancy of the survival of the patients (Nakamura et al., 1997). 
Table 2. TP53 gene analysis results

\begin{tabular}{lcc} 
Sample & TP53 mutation & Exon/intron \\
\hline Primary tumor & Arg156-Pro & 5 \\
& $+4 b p ; c-t$ & Intron 9 \\
Rec. 1a & Arg156-Pro & 5 \\
& $+4 b p ; c-t$ & Intron 9 \\
Rec. 1b & Thr211-lle & 6 \\
Rec. 2 & Arg156-Pro & 5 \\
& -32bp; ins c & Intron 2 \\
\hline
\end{tabular}

Abbreviations: $+b p$, base pairs from splice site donor site; -bp, base pairs from splice site acceptor site.

In our patient, with a long clinical history, the first tumor resection material showed 2 different components, one with low cellularity and one with increased cellularity and pleomorphism, although with insufficient evidence for full malignancy, and both with low MIB LI. The tumor rapidly progressed to a full blown malignancy with high mitotic activity and high MIB LI. The karyotype after radiotherapy was very complex, with at least 3 different clones, together with multiple balanced chromosomal rearrangements; whereas in the first recurrence, before radiotherapy, fewer different clones and no balanced chromosomal abnormalities could be found. It is known that therapeutic irradiation can induce solid tumors or perhaps change the character of already existing tumor tissue. Dirks et al. (1994) described 6 children who developed anaplastic astrocytomas after receiving radiation therapy for low-grade astrocytomas. Chauveinc et al. (1997) concluded that the presence of multiple chromosomal rearrangements, principally of the balanced type, and multiclonality strongly suggest the radiationinduced nature of 3 meningiomas in a single patient. So these 2 characteristics - multiclonality and high frequency of balanced chromosomal rearrangements - might be the result of radiation-induced changes in the already existing tumor in this patient.

Pediatric low-grade astrocytomas tend to yield normal karyotypes or only numerical chromosomal aberrations, whereas most complex karyotypes with structural abnormalities are found in more aggressive tumors like anaplastic astrocytomas and glioblastomas multiforme, though without a single characteristic anomaly (Bhattacharjee et al., 1997; Biegel, 1997; Bigner et al., 1997; Fujii et al., 1994; Griffin et al., 1992; Karnes et al., 1992; Neumann et al., 1993; Sainati et al., 1996; Thiel et al., 1993; Wernicke et al., 1997). Chromosome 1 abnormalities are frequently seen in pediatric tumors of the CNS (Douglass et al., 1985; Sainati et al., 1996; Thiel et al., 1993). Sainati et al. (1996) suggested a possible association between chromosome 1 rearrangements and very aggressive childhood CNS tumors, regardless of the primary tumor histology. They also found complex karyotypes and pseudopolyploidy frequently associated with a poor prognosis.

As to a possible relation between prognosis and mutation of the TP53 gene, according to the literature, TP53 gene mutations appear to be an early event in the genesis of astrocytomas, at least in adults. Sidransky et al. (1992) suggested that mutation of TP53 is an important step in

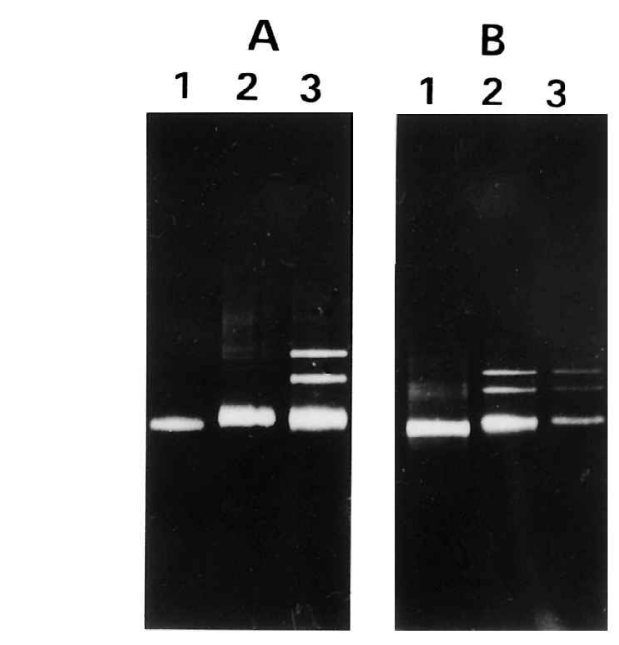

Fig. 4. DGGE aberrant banding pattern for the possibly causative TP53 mutation occurring in exon 5 of the TP53 gene. In both A and $B$, the DGGE banding pattern is representative of a control (lane 1), Rec. 1a (lane 2), and primary tumor (lane 3). In A, Rec. 1a appears as a hemizygous/homozygous single DGGE band. In B, Rec. 1a was mixed with control DNA before heteroduplexing and therefore gave the same banding pattern as heterozygous primary tumor.

the progression from low- to high-grade astrocytomas in adults. However, mutations of this gene are rare in both pilocytic and low-grade astrocytomas of childhood (Felix et al., 1995; Litofsky et al., 1994; Orellana et al., 1998), and there are hardly any data about progression of such tumors in relation to TP53 mutation. Pollack et al. (1997) found TP53 mutation or p53 immunopositivity in highgrade pediatric astrocytomas of the brainstem to be associated with poor survival. In low-grade adult astrocytomas, p53 immunopositivity seems to be related to a worse prognosis (Chozick et al., 1994; Iuzzolino et al., 1994). A previous study by Dirven (1998), enlarged to include a series of 20 pilocytic (World Health Organization grade I) and 2 low-grade (World Health Organization grade II) astrocytomas, showed TP53 probably to be involved in these tumor types (Hayes et al., 1999b). The 4 tumors described in that series of patients with progression of residual tumor after postoperative follow-up, none of which had a TP53 mutation, were all of the pilocytic type. Both low-grade astrocytomas (one with and one without a TP53 mutation) were totally resected and, therefore, progression could not be assessed. A relationship with tumor behavior thus remains unclear. In retrospect, the unexpected aggressive behavior of the low-grade, nonpilocytic astrocytoma described in this study might have been predicted from the highly abnormal karyotype, together with the clonal abnormality of chromosome 1 , found before radiation therapy.

\section{Acknowledgments}

The authors would like to thank A.Y. van der Veen for performing FISH analysis and E. Verlind for sequencing DNA samples. S. Noorman processed the photomicrographs. 


\section{References}

Bhattacharjee, M.B., Armstrong, D.D., Vogel, H., and Cooley, L.D. (1997) Cytogenetic analysis of 120 primary pediatric brain tumors and literature review. Cancer Genet. Cytogenet. 97, 39-53.

Biegel, J.A. (1997) Genetics of pediatric central nervous system tumors. J. Pediatr. Hematol. Oncol. 19, 492-501.

Bigner, S.H., McLendon, R.E., Fuchs, H., McKeever, P.E., and Friedman, H.S. (1997) Chromosomal characteristics of childhood brain tumors. Cancer Genet. Cytogenet. 97, 125-134.

Chauveinc, L., Ricoul, M., Sabatier, L., Gaboriaud, G., Srour, A., Bertagna, X., and Dutrillaux, B. (1997) Dosimetric and cytogenetic studies of multiple radiation-induced meningiomas for a single patient. Radiother. Oncol. 43, 285-288.

Chozick, B.S., Pezzullo, J.C., Epstein, M.H., and Finch, P.W. (1994) Prognostic implications of $\mathrm{p} 53$ overexpression in supratentorial astrocytic tumors. Neurosurgery 35, 831-837.

Cohen, M.E., and Duffner, P.K. (1994) Brain Tumors in Children. Principles of Diagnosis and Treatment.Second edition. International Review of Child Neurology Series. New York: Raven Press Books Ltd. p. 203-218.

Dirks, P.B., Jay, V., Becker, L.E., Drake, J.M., Humphreys, R.P., Hoffman, H.J., and Rutka, J.T. (1994) Development of anaplastic changes in low-grade astrocytomas of childhood. Neurosurgery 34, 68-78.

Dirven, C.M.F., Hayes, V.M., Koudstaal, J., Molenaar, W.M., and Mooij, J.J.A. (1998) TP53 is involved in pilocytic astrocytomas but has no relation with tumor behavior. In: Dirven, C.M.F. The Pilocytic Astrocytoma, Immunohistochemical and Genetic Studies in Relation to Tumor Behavior. Thesis Rijksuniversiteit Groningen. p. 97-110.

Douglass, E.C., Green, A.A., Hayes, F.A., Etcubanas, E., Horowitz, M., and Wilimas, J.A. (1985) Chromosome 1 abnormalities: A common feature of pediatric solid tumors. J. Natl. Cancer Inst. 75, 51-54.

Felix, C.A., Slavc, I., Dunn, M., Strauss, E.A., Phillips, P.C., Rorke, L.B., Sutton, L., Bunin, G.R., and Biegel, J.A. (1995) P53 gene mutations in pediatric brain tumors. Med. Pediatr. Oncol. 25, 431-436.

Fujii, Y., Hongo, T., and Hayashi, Y. (1994) Chromosome analysis of brain tumors in childhood. Genes Chromosomes Cancer 11, 205-215.

Giannini, C., and Scheithauer, B.W. (1997) Classification and grading of lowgrade astrocytic tumors in children. Brain Pathol. 7, 785-798.

Griffin, C.A., Long P.P., Carson, B.S., and Brem, H. (1992) Chromosome abnormalities in low-grade central nervous system tumors. Cancer Genet. Cytogenet. 60, 67-73.

Hayes, V.M., Bleeker, W., Verlind, E., Timmer, T., Karrenbeld, A., Plukker, J.T., Marx, M.P., Hofstra, R.M., and Buys, C.H. (1999a) Comprehensive TP53denaturing gradient gel electrophoresis mutation detection assay also applicable to archival paraffin-embedded tissue. Diagn. Mol. Pathol. 8, 2-10.

Hayes, V.M., Diven, C.M., Dam, A., Verlind, E., Molenaar, W.M., Mooij, J.J., Hofstra, R.M., and Buys, C.H. (1999b)

High frequency of TP53 mutations in juvenile pilocytic astrocytomas indicates role of TP53 in the development of these tumors. Brain Pathol. 9, 463467.

Hebsgaard, S.M., Korning, P.G., Tolstrup, N., Engelbrecht, J., Rouze, P., and
Brunak, S. (1996) Splice site prediction in Arabidopsis thaliana pre-mRNA by combining local and global sequence information. Nucleic Acids Res. 24, 3439-3452.

Iuzzolino, P., Ghimenton, C., Nicolato, A., Giorgiutti, F., Fina, P., Doglioni, C., and Barbareschi, M. (1994) P53 protein in low-grade astrocytomas: A study with long-term follow-up. Br. J. Cancer. 69, 586-591.

Karnes, P.S, Tran, T.N., Cui, M.Y., Raffel, C., Gilles, F.H, Barranger, J.A., and Ying, K.L. (1992) Cytogenetic analysis of 39 pediatric central nervous system tumors. Cancer Genet. Cytogenet. 59, 12-19.

Lapras, C., Patet, J.D., Lapras, C., Jr., and Mottolese, C. (1986) Cerebellar astrocytomas in childhood. Childs Nerv. Syst. 2, 55-59.

Litofsky, N.S., Hinton, D., and Raffel, C. (1994) The lack of a role for p53 in astrocy tomas in pediatric patients. Neurosurgery 34, 967-972.

Nakamura, M., Konishi, N., Tsunoda, S., Hiasa, Y., Tsuzuki, T., Inui, T., and Sakaki, T. (1997) Retinoblastoma protein expression and MIB-1 correlate with survival of patients with malignant astrocytoma. Cancer 80, 242-249.

Neumann, E., Kalousek, D.K., Norman, M.G., Steinbok, P., Cochrane, D.D., and Goddard, K. (1993) Cytogenetic analysis of 109 pediatric central nervous system tumors. Cancer Genet. Cytogenet. 71, 40-49.

Orellana, C., Hernandez-Marti, M., Martinez, F., Castel, V., Millan, J.M., Alvarez Garijo, J.A., Prieto, F., and Badia, L. (1998) Pediatric brain tumors: Loss of heterozygosity at 17p and TP53 gene mutations. Cancer Genet. Cytogenet. 102, 93-99.

Pollack, I.F., Hamilton, R.L., Finkelstein, S.D., Campbell, J.W., Martinez, A.J., Sherwin, R.N., Bozik, M.E., and Gollin, S.M. (1997) The relationship between TP53 mutations and overexpression of p53 and prognosis in malignant gliomas of childhood. Cancer Res. 57, 304-309.

Sainati, L., Bolcato, S., Montaldi, A., Celli, P., Stella, M., Leszl, A., Silvestro, L., Perilongo, G., Cordero di Montezemolo, L., and Basso, G. (1996) Cytogenetics of pediatric central nervous system tumors. Cancer Genet. Cytogenet. 91, 13-27.

Sidransky, D., Mikkelsen, T., Schwechheimer, K., Rosenblum, M.L., Cavanee, W., and Vogelstein, B. (1992) Clonal expansion of p53 mutant cells is associated with brain tumour progression. Nature 355, 846-847.

Sgouros S., Fineron P.W., and Hockley, A.D. (1995). Cerebellar astrocytoma of childhood: Long-term follow-up. Childs Nerv. Syst. 11, 89-96.

Standing Committee on Human Cytogenetic Nomenclature (1995) An International System for Human Cytogenetic Nomenclature. Basel: S. Karger.

Stein, A., and Raoult, D. (1992) A simple method for amplification of DNA from paraffin-embedded tissues. Nucleic Acids Res. 20, 5237-5238.

Thiel, G., Lozanova, T., Vogel, S., Kintzel, D., Janisch, W., and Witkowski, R. (1993) Age-related nonrandom chromosomal abnormalities in human low-grade astrocytomas. Hum. Genet. 91, 547-550.

Vindelov, L.L., Christensen, I.J., and Nissen, N.I. (1983) A detergent-trypsin method for the preparation of nuclei for flow cytometric DNA analysis. Cytometry 3, 323-327.

Wernicke, C., Thiel, G., Lozanova, T., Vogel, S., and Witkowski, R. (1997) Numerical aberrations of chromosomes 1, 2, and 7 in astrocytomas studied by interphase cytogenetics. Genes Chromosomes Cancer 19, 6-13. 